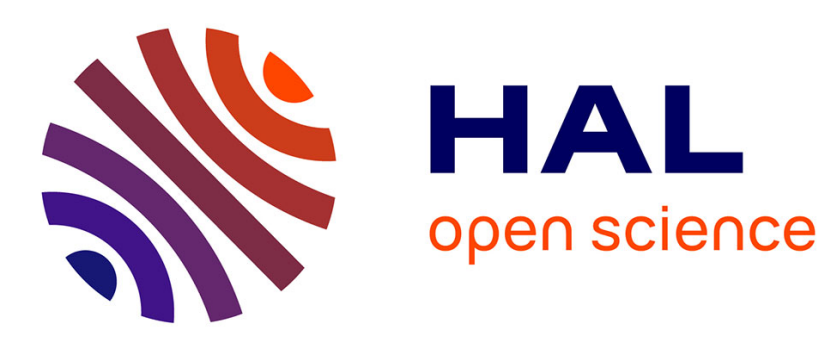

\title{
La difficile institutionnalisation d'une GRH territoriale. Une étude de cas exploratoire
}

Nicolas Arnaud, Stéphane Fauvy, Hadj Nekka

\section{To cite this version:}

Nicolas Arnaud, Stéphane Fauvy, Hadj Nekka. La difficile institutionnalisation d'une GRH territoriale. Une étude de cas exploratoire. Revue Française de Gestion, 2013, 232, pp.15-33. 10.3166/rfg.231.1533 . hal-00837590

\section{HAL Id: hal-00837590 \\ https://hal-audencia.archives-ouvertes.fr/hal-00837590}

Submitted on 23 Jun 2013

HAL is a multi-disciplinary open access archive for the deposit and dissemination of scientific research documents, whether they are published or not. The documents may come from teaching and research institutions in France or abroad, or from public or private research centers.
L'archive ouverte pluridisciplinaire HAL, est destinée au dépôt et à la diffusion de documents scientifiques de niveau recherche, publiés ou non, émanant des établissements d'enseignement et de recherche français ou étrangers, des laboratoires publics ou privés. 


\title{
La difficile institutionnalisation d'une politique de GRH au sein d'un pôle de compétitivité : Une étude de cas exploratoire
}

\author{
Nicolas ARNAUD (Audencia Nantes - Ecole de Management) \\ nicolasarnaud@audencia.com \\ Stéphane FAUVY (ESSCA - School of Management) \\ stephane.fauvy@essca.fr \\ Hadj NEKKA (LUNAM Université, Université d'Angers, \\ GRANEM ) \\ hadj.nekka@univ-angers.fr
}

\begin{abstract}
Résumé : Cet article s'intéresse au développement d'une politique de GRH au niveau d'un pôle de compétitivité. Elle s'appuie sur une recherche empirique de type qualitatif effectuée au sein d'un pôle de compétitivité spécialisé dans le végétal. En s'appuyant sur des travaux issus de l'approche discursive des institutions, ce papier montre la difficile mise en place d'une politique de GRH, matérialisée par des outils et des processus RH mutualisés, au sein du pôle de compétitivité étudié. Au-delà de la mise en évidence de visions concurrentes quant au rôle des ressources humaines au niveau inter organisationnel, ce travail cherche à identifier les leviers d'action permettant de suivre les évolutions et la structuration d'une GRH territoriale.
\end{abstract}

Mots clé : pôle de compétitivité, institutionnalisation, discours, GRH, réunions.

Asbtract: This article investigates the development of a policy of HR Management within a French cluster. It is based on a qualitative-type research conducted within a cluster specialized in plants. By referring to work studying the discursive approach to institutions, we show the difficulty of establishing a common HRM policy, based on shared HR tools and processes, within the cluster. Beyond the identification of competing visions of the role of human resources at the inter-organizational level, our work seeks to identify the levers that will enable to monitor the development and structuring of a territorial HRM.

Key words: French cluster, institutionalization, discourse, HRM, meetings. 
En 2005, l'Etat français lance la création des pôles de compétitivité. Ces pôles rassemblent des organismes de recherche, des entreprises, des universités et des collectivités locales dont l'objectif est de favoriser l'innovation sur un secteur économique. Selon leur principe fondateur, les pôles ont été élaborés entre des partenaires très différents et rassemblent donc des salariés relevant de modèles de gestion des ressources humaines (GRH) variés. Plusieurs voix, parmi lesquelles des représentants des pouvoirs publics mais aussi des chercheurs et des praticiens, s'élèvent aujourd'hui pour attirer l'attention sur cette dimension humaine (Bertrand, et al., 2008, Colle, et al., 2009, Defélix, et al., 2008).

L'état des connaissances sur les pôles de compétitivité est à un stade embryonnaire et ceci malgré l'existence d'une littérature relative au management des collaborations interorganisationnelles et une autre abordant notamment les conditions et les pratiques de la gestion des projets (Defelix, et al., 2008). Au-delà des critiques associées à ces approches théoriques (Defélix, et al., 2008), l'une comme l'autre traite des enjeux humains. Cependant, ces enjeux ont été peu étudiés au sein des pôles de compétitivité. Ainsi, quelques contributions ont essayé d'y remédier même si elles restent à un stade exploratoire (Culié, et al., 2006, Defélix, et al., 2008, Mazzilli, 2010). Désormais, repenser la GRH en dehors des frontières de l'organisation et au sein des réseaux organisationnels fait actuellement l'unanimité, mais toute la question reste celle de sa concrétisation (Mazzilli, 2010). Si les chercheurs ont réussi à bien cerner les caractéristiques des dispositifs de GRH territoriale (variété de leur forme, diversité des structures porteuses de ces dispositifs, leur incorporation dans diverses instrumentations de GRH inter-organisationnelles), la grille théorique permettant de mieux saisir leur construction reste néanmoins à consolider. Ce domaine de recherche - entre GRH Territoriale et GRH dans les pôles de compétitivité - se caractérise en effet par la pluralité des ancrages théoriques : la théorie des parties prenantes (Bories-Azeau, et al., 2007, Bourgain et Tixier, 2010, Loubès et Bories-Azeau, 2011), l'économie de la proximité (Barabel, et al., 2009), de l'Actor Network Theory (Mazzilli, 2009, Mazzilli, 2010), 
voire la création de grilles de lecture combinant plusieurs approches théoriques (Calamel, et al., 2011, Defélix, et al., 2010). Cette hétérogénéité traduit le constat de Loubès et BoriesAzeau (2011), selon lequel il n'existe pas encore à ce jour de théorie stabilisée de la GRH territoriale. Dans ce contexte, connaître en profondeur les dynamiques d'émergence de ces dispositifs passe nécessairement par la mobilisation d'un cadre théorique pertinent. Malgré l'intérêt incontestable de ces travaux pour parfaire notre connaissance d'une GRH territoriale, certains aspects restent à approfondir. D'une part, ces travaux tendent à minimiser la place des contributions individuelles dans l'élaboration de dispositifs $\mathrm{RH}$ et certains éléments de contexte n'apparaissent pas toujours clairement comme facteur de structuration des actions RH développées (Calamel, et al., 2011). D’autre part, s’ils montrent la diversité de ces dernières, ces travaux n'abordent pas explicitement la question de leur cohérence au niveau du pôle. Partant de ce constat, cet article entend apporter des éléments permettant de suivre l'émergence d'une GRH inter-organisationnelle. Plus précisément, l'objet de cette recherche est d'étudier les actions permettant l'institutionnalisation d'une politique de GRH au sein d'un pôle de compétitivité. Pour atteindre cet objectif, nous mobilisons les travaux de Phillips et al. (2004), qui proposent une théorie discursive du processus d'institutionnalisation. Trois raisons justifient ce choix. La première concerne la prise en compte de la dimension temporelle et processuelle du phénomène étudié, la deuxième autorise à considérer l'appareillage gestionnaire comme des incarnations discursives (le discours ne devant pas être restreint à la parole). Enfin, la troisième a trait aux enjeux de l'ingénierie des dispositifs territoriaux qui sont tels que les personnes importent plus que les outils car tout est à construire et à légitimer (Bories-Azeau, et al., 2007). La présentation des travaux se référant à l'approche discursive des institutions fera l'objet de notre première partie. La méthodologie de la recherche sera ensuite exposée avant de proposer l'analyse du cas exploratoire que constitue VEGEVALLEY, pôle de compétitivité à vocation mondiale spécialisé dans le végétal.

\section{INSTITUTIONNALISATION ET DISCOURS}




\subsection{Approche discursive des organisations}

L'approche discursive des organisations met en avant la dimension communicationnelle des organisations (Grant, et al., 1998). Ces dernières apparaissent et évoluent en fonction des actions quotidiennes des acteurs qui produisent des textes, c'est-à-dire «des artefacts symboliques écrits et oraux » (Robichaud, et al., 2004). Ces actions sont susceptibles d'être réalisées dans des cadres plus ou moins formalisés comme des activités quotidiennes, des groupes de travail, des instances de décision. Plus le cadre d'occurrence est formalisé, plus les textes produits seront objectivés (mots, énonciation des acteurs versus diagrammes, rapports, tableaux).

Les actions des acteurs produisent donc différentes formes de textes. Pour Detchessahar et Journé (2007), les outils de gestion, les métaphores, les récits, les écrits, les diagrammes, etc. sont autant d'exemples de cette pluralité. La réalité d'une organisation, qu'elle soit plus ou moins délimitée par des frontières (comme les entreprises) ou émergente (comme les groupes de travail organisés par le pôle étudié pour élaborer la réflexion et l'action RH), est toujours constituée par un ensemble de textes (Grant, et al., 2004). Cet ensemble constitue alors un discours organisationnel plus ou moins cohérent et potentiellement, étape ultime, une institution. Notre travail empirique ici n'a pas consisté à vérifier la portée de cette théorie. En revanche, la relation développée entre actions, textes, discours et institution apparaît pertinente pour exploiter nos données empiriques relatives au processus d'institutionnalisation des pratiques RH. En effet, il ressort de l'enquête que les outils RH ont participé à la construction de textes qui s'intègrent plus ou moins facilement dans un discours unique et cohérent tentant d'institutionnaliser une politique RH au niveau du pôle.

En cela, les travaux de Phillips et al. (2004) apportent un éclairage intéressant. A partir de la compréhension des formes du discours organisationnel, ces travaux permettent d'explorer plus en détail les dynamiques d'institutionnalisation. Les notions d'action, de textes et de discours deviennent alors les concepts permettant d'appréhender une telle dynamique. 
L'analyse faite par Phillips et al. (2004) des travaux célèbres de Palmer, Jennings et Zhou

(1993) sur l'institutionnalisation de la structure multidivisionnelle illustre la proposition

théorique en montrant le rôle central des textes, de même que le lien entre discours et

institutions.

Tableau 1 : L'exemple de la structure multidivisionnelle (d'après Phillips et al., 2004)

\begin{tabular}{|c|c|}
\hline $\begin{array}{c}\text { 1. La production et la diffusion de textes sont } \\
\text { associées aux pressions isomorphiques jouant } \\
\text { un rôle dans le processus } \\
\text { d'institutionnalisation }\end{array}$ & $\begin{array}{l}\text { 2. Les pratiques et compréhensions } \\
\text { institutionnelles constituant la forme } \\
\text { multidivisionnelle sont les produits } \\
\text { du discours }\end{array}$ \\
\hline $\begin{array}{l}\text { - Les pressions normatives ont opéré au sein de } \\
\text { la formation de cadres seniors à l'école de commerce } \\
\text { d'élite, dans laquelle l'utilisation du livre de Chandler } \\
\text { (1962) sur la forme multidivisionnelle était } \\
\text { particulièrement importante. Ce texte particulier, } \\
\text { comme les innombrables lectures et séminaires dans } \\
\text { les écoles de commerces américaines basés sur celui- } \\
\text { ci, ont influencé l'institutionnalisation de la forme } \\
\text { multidivisionnelle. } \\
\text { - Les pressions mimétiques ont découlé des } \\
\text { directions imbriquées qui ont réuni des directeurs de } \\
\text { différentes entreprises. Tandis que ces directeurs ont } \\
\text { été témoins de certains aspects de la forme } \\
\text { multidivisionnelle directement, la plus grande part de } \\
\text { leurs connaissances concernant leur propre entreprise, } \\
\text { et particulièrement les autres entreprises, viendrait de } \\
\text { textes tels que graphiques organisationnels, rapports, } \\
\text { conversations, histoires et ainsi de suite. } \\
\text { - Les grandes entreprises exerçaient des } \\
\text { pressions coercitives sur des partenaires pour qu'ils } \\
\text { adoptent la même structure car cela rendait plus facile } \\
\text { d'obtenir et d'évaluer l'information provenant } \\
\text { d'individus dans des rôles organisationnels analogues, } \\
\text { tandis que les banques utilisaient le pouvoir basé sur la } \\
\text { propriété pour mettre sous pression les entreprises afin } \\
\text { qu'elles adoptent la forme multidivisionnelle car elle } \\
\text { rendait plus facile la diversification, en minimisant le } \\
\text { risque et la probabilité de défaut de paiement. Ces } \\
\text { pressions coercitives auraient été relayées par des } \\
\text { textes, tels que graphiques, rapports, comptabilité etc. } \\
\text { - L'institutionnalisation de la forme multi- } \\
\text { divisionnelle ne s'est pas produite car les acteurs dans } \\
\text { les diverses organisations l'observaient directement en } \\
\text { action, mais à cause de l'accumulation de textes } \\
\text { commerciaux, professionnels et académiques qui } \\
\text { l'expliquaient, la légitimaient, la validaient et la } \\
\text { promouvaient. }\end{array}$ & $\begin{array}{l}\text { - Les nombreux textes discutant } \\
\text { l'efficacité de cette forme, expliquant son } \\
\text { utilisation par des entreprises leaders et } \\
\text { présentant l'approbation d'universitaires et } \\
\text { de leaders économiques, ainsi que toute une } \\
\text { gamme d'autres textes, ont généré le large } \\
\text { discours de la structure multidivisionnelle. } \\
\text { Il existait des recueils de textes qui ont } \\
\text { dessiné des compréhensions très répandues } \\
\text { de ce que comprenait la structure } \\
\text { multidivisionnelle. } \\
\text { - Les managers pensaient de plus en } \\
\text { plus à organiser leurs entreprises de cette } \\
\text { façon et instituaient des changements en } \\
\text { accord avec elle, le discours donna une } \\
\text { réalité à cette structure. Au fil du temps, le } \\
\text { discours a constitué la structure } \\
\text { multidivisionnelle en tant qu'institution car } \\
\text { ne pas l'adopter engendrait des coûts } \\
\text { croissants. Cela conduisait à un un } \\
\text { questionnement sur la légitimité soulevée } \\
\text { par les raisons potentielles pour lesquelles } \\
\text { l'entreprise n'adoptait pas la pratique } \\
\text { acceptée (coûts sociaux), et cela a conduit } \\
\text { les banques à ne pas vouloir investir ou } \\
\text { d'autres entreprises à ne pas vouloir } \\
\text { collaborer (coûts économiques). En plus, en } \\
\text { comparaison à l'adoption d'une structure } \\
\text { «toute faite », il faudrait une quantité } \\
\text { importante de réflexion et d'effort pour } \\
\text { concevoir et mettre en place une structure } \\
\text { alternative (coûts cognitifs). } \\
\text { - Le discours de la structure } \\
\text { multidivisionnelle a formé une institution, } \\
\text { aboutissant à une action dans un large } \\
\text { champ institutionnel au fur et à mesure que } \\
\text { les sociétés adoptaient cette structure } \\
\text { approuvée et légitime. }\end{array}$ \\
\hline
\end{tabular}

\subsection{La dynamique discursive des processus d'institutionnalisation}


Ce cadre théorique facilite la compréhension des difficultés relatives à l'institutionnalisation des pratiques RH. Le mécanisme central est le suivant : les actions des acteurs génèrent des textes s'encastrant dans un discours susceptible de produire une institution qui contraint et habilite en retour les actions. Trois problématiques rendent compte de ce processus: qui produit les textes? Quelles formes prennent-ils? Quelle est la cohérence du discours organisationnel ?

Tout d'abord, la problématique organisationnelle déclenchant l'action, et donc le processus de production de textes, amène les acteurs à en construire le sens ainsi que sa légitimité. Autrement dit, la question de l'innovation sociale au sein d'un pôle doit être perçue comme une nécessité par les acteurs impliqués plutôt qu'une obligation. A cet égard, Hardy et Phillips (2004) proposent un ensemble de facteurs concernant les caractéristiques du producteur du texte. En effet, pour être reconnu comme agent légitime, le producteur du texte doit s'assurer que son «droit à parler» soit reconnu. En d'autres termes, le producteur du texte doit être capable de faire «adhérer » au texte par des moyens plus ou moins coercitifs ou d'en ajouter en raison de sa position centrale au sein d'un réseau d'organisations par exemple. Une telle position lui permet plus facilement de disséminer ses textes à un grand nombre d'acteurs (Wasserman et Galaskiewicz, 1994). Ensuite les textes possèdent des différences dans les processus et les caractéristiques de leur production, ce qui minimise leur chance de s'intégrer au discours organisationnel. La forme du texte, et notamment son degré d'objectivation, peut influencer la possibilité d'être utilisée par d'autres organisations, tout comme la relation d'un texte à un autre (Hardy et Phillips, 2004). Plus cette intertextualité est importante et plus le discours gagnera en cohérence, puisqu'évoquant des représentations et significations déjà largement ancrées. Enfin, la probabilité d'un discours de produire une institution dépend de nombreux facteurs, dont l'un concerne la construction interne du discours lui-même. Certains discours sont plus cohérents que d'autres, c'est-à-dire que les divers textes convergent ou non dans leurs descriptions et explications de l'aspect particulier de la réalité sociale. 
Detchessahar et Journé (2007) montrent par exemple comment deux outils de gestion (une matrice de compétences et des grilles de poly compétences) entrent en conflit, rendant au final difficile l'élaboration d'un discours organisationnel cohérent et unique. De plus, certains discours sont plus structurés que d'autres, les textes qui les constituent s'articulant de manière plus facilement compréhensible. Les discours cohérents et structurés présentent une vision plus unifiée de certains aspects de la réalité sociale, qui s'en trouve ainsi réifiée et considérée comme acquise. Plus une construction sociale est acquise, plus il est difficile et coûteux d'adopter des comportements qui ne sont pas en accord avec elle (Phillips, et al., 2004). Enfin, la probabilité d'un discours de produire des institutions puissantes dépendra du degré auquel le discours est en accord avec des discours plus larges et l'existence de discours concurrents. Les auteurs entendent par discours concurrents un autre ensemble de textes interdépendants proposant des constructions sociales alternatives du même aspect de la réalité sociale. L'habilité politique des dirigeants apparaît alors intéressante à développer dans la mesure où le pouvoir dont ils disposent et les actions politiques qu'ils peuvent mettre en place sont une condition, parmi d'autres, du processus d'institutionnalisation.

\section{Méthodologie}

Cette recherche exploratoire, justifié par le caractère émergent de notre objet, répond à une problématique provenant tant du monde académique que professionnel notamment formulée par le pôle : comment innover en matière de GRH au sein d'un pôle de compétitivité spécialisé dans le végétal ? Le recours à l'étude de cas (Yin, 2003) a permis d'étudier un phénomène empirique encastré dans des problématiques de territoire spécifiques. En nous appuyant sur les travaux de Stake (2005), le cas rapporté dans cette recherche a été étudié à la fois de manière intrinsèque (intérêt en soi, analyse de la problématique $\mathrm{RH}$ du pôle) et instrumentale (utilisé pour discuter la pertinence la théorie de Philips et al., 2004). L'équipe de trois chercheurs a pu évoluer au sein du pôle et de quelques entreprises membres en tant qu'observateurs extérieurs. 
Le corpus des données de terrain a été construit de manière classique à l'aide d'une triangulation croisant plusieurs techniques de recueil de données animée par les trois chercheurs :

- 15 entretiens semi-directifs avec des directeurs de site, des managers (directeur des ressources humaines, directeur commercial, directeur production), des consultants, des responsables du pôle.

- 3 «réunions stratégiques » visant à développer la réflexion RH ont pu être observées auxquelles participaient des employés du pôle, des enseignants, des représentants de collectivités publiques, deux groupements d'employeurs et quatre à six entreprises de production (sur 20 personnes présentes en moyenne). Dix personnes présentes à ces réunions ont pu être interviewées.

- la collecte de documents relatifs aux pratiques actuelles en termes de RH et aux actions envisagées à la suite des réunions RH (compte rendu de réunion, contrat de performance, lettre d'information, etc.).

Les observations ont fait l'objet d'un traitement qualitatif à l'aide d'une analyse de contenu. Les entretiens ont été réalisés en deux temps durant le premier semestre de l'année 2010. Il s'agissait d'abord de reconstruire a posteriori les étapes de la réflexion $\mathrm{RH}$ pour les réunions antérieures au commencement de notre recherche. Pour cela, nous avons rencontré des acteurs présents aux différentes réunions afin d'entendre leur version ainsi que les conséquences des décisions prises pour le fonctionnement de leur organisation d'appartenance. Une seconde phase a consisté à rencontrer des responsables d'entreprises productives des différentes filières du pôle. Nous avons ainsi pu recueillir des informations sur les transformations à l'œuvre dans leur secteur ainsi que sur les difficultés rencontrées en termes de GRH.

\section{ETUDE DE CAS : LA DIFFICILE CONSTRUCTION D'UNE POLITIQUE RH AU SEIN D'UN PÔLE DE COMPÉTITIVITÉ}

\subsection{VEGEVALLEY : un pôle de compétitivité à vocation mondiale}


Le pôle de compétitivité VEGEVALLEY a pour objectif de devenir une référence mondiale de l'innovation dans le végétal. VEGEVALLEY regroupe huit filières du végétal spécialisé, défini comme une production végétale à haute valeur ajoutée, caractérisée par un besoin intensif en facteurs de production (main d'œuvre, équipements) et générant un chiffre d'affaires élevé au mètre carré. Le tableau suivant présente les différentes filières représentées au sein de VEGEVALLEY.

Tableau 2 : Les huit filières du végétal spécialisé de VEGEVALLEY

\begin{tabular}{|c|c|}
\hline L'horticulture & $\begin{array}{l}\text { - 1er rang national pour les plantes en pots, les plantes à massif, les jeunes } \\
\text { plants de pépinière, les bulbes, le dahlia, les arbustes en conteneur. } \\
\text { - } 90 \% \text { de la production destinée aux marchés local et national, } 10 \% \text { de la } \\
\text { production exportée essentiellement en Europe. } \\
\text { - } 3400 \text { hectares cultivés, } 280 \text { entreprises pour } 3300 \text { emplois permanents. }\end{array}$ \\
\hline Le maraîchage & $\begin{array}{l}\text { - Deux légumes leaders : la mâche nantaise avec } 80 \% \text { de la production } \\
\text { nationale et le poireau primeur avec } 27 \% \text {. } \\
\text { - } 20 \text { à } 30 \% \text { du chiffre d'affaires réalisé à l'export. } \\
\text { - } 41 \% \text { des entreprises groupées en coopératives. } \\
\text { - } 20200 \text { salariés soit } 4300 \text { ETP. }\end{array}$ \\
\hline L'arboriculture & $\begin{array}{l}\text { - 1er rang national pour la pomme et le cassis. } \\
\text { - } 50 \% \text { des volumes exportés produits en Val de Loire. } \\
\text { - } 471 \text { entreprises et } 22700 \text { emplois. }\end{array}$ \\
\hline La viticulture & $\begin{array}{l}-3^{\text {ème }} \text { vignoble français } \\
-75 \% \text { de la production est consommée en France, } 25 \% \text { exportée en Grande } \\
\text { Bretagne, Belgique, Allemagne, Pays Bas et Etats Unis. } \\
\text { - } 42000 \text { hectares de vignoble, } 4100 \text { exploitations, } 2000 \text { salariés permanents. }\end{array}$ \\
\hline Les semences & $\begin{array}{l}\text { - 1er rang national pour les semences potagères et florales avec } 25 \% \text { de la } \\
\text { production nationale, } 60 \text { millions } € \text { de chiffre d'affaires. } \\
\text { - 1er producteur européen de semences en volume, 3e exportateur mondial. } \\
\text { - Plus de } 1330 \text { exploitations pour près de } 3000 \text { emplois. }\end{array}$ \\
\hline $\begin{array}{l}\text { Les plantes « Santé - } \\
\text { Beauté - Bien-être » }\end{array}$ & $\begin{array}{l}\text { - 1er rang national pour les plantes médicinales avec plus de } 70 \text { espèces } \\
\text { cultivées sur } 700 \text { hectares. } \\
\text { - Plus de } 20 \text { entreprises de production, transformation et négoce, leaders } \\
\text { nationaux et internationaux implantés en Pays de Loire. } \\
\text { - Marchés concernés : l'alimentation, la cosmétique, la santé des hommes, des } \\
\text { animaux, des plantes. }\end{array}$ \\
\hline Les champignons & $\begin{array}{l}\text { - 1ère région française. } 50 \% \text { de la production française cultivée en Maine et } \\
\text { Loire avec le produit leader « Champignon de Paris ». } \\
\text { - } 875000 \text { tonnes dont } 42 \% \text { destinés à l'industrie et } 58 \% \text { au frais. }\end{array}$ \\
\hline Le cidre et le tabac & $\begin{array}{l}-13 \% \text { de la production nationale de cidre. } \\
\text { - } 535 \text { hectares dédiés à l'exploitation de tabac. }\end{array}$ \\
\hline
\end{tabular}

Impulsé par l'Etat français, VEGEVALLEY a annoncé en 2009 la signature d'un nouveau Contrat de Performance. VEGEVALLEY doit donc engager une politique de développement sur la période 2009/2011 qui répond à deux objectifs principaux : favoriser la création et la production de végétaux favorables à la biodiversité, à l'environnement et à la santé, et ouvrir 
de nouveaux marchés aux entreprises du pôle grâce à des végétaux innovants. Pour réaliser ces deux objectifs, VEGEVALLEY est amené à définir comme axe stratégique la construction d'une politique de gestion des RH. De manière non exhaustive, il est possible d'évoquer l'attractivité des métiers (comment attirer les «hauts profils» en Anjou ?), le recrutement et la fidélisation des salariés liés à la saisonnalité de l'activité (quels messages construire pour sensibiliser les jeunes, comment prendre en compte les besoins des entreprises ?), la sécurité au travail, etc.

\subsection{Etude du processus d'institutionnalisation}

\subsubsection{Les Rendez-vous stratégiques $R H$}

La première réunion stratégique sur le thème des $\mathrm{RH}$ a constitué une étape particulièrement importante. Cet atelier RH s'est tenu en octobre 2008 et a été l'occasion d'engager la réflexion autour d'une question centrale : à quoi pourrait ressembler une politique de GRH au niveau de VEGEVALLEY? Autrement dit, lors de cette réunion, les acteurs ont pris collectivement conscience du besoin de construire le sens et la forme d'une telle politique RH. La politique RH de VEGEVALLEY est devenue une question légitime. Le caractère légitime de cette question apparaît sous plusieurs aspects. D'un côté, elle émerge comme un objectif nouveau donné aux pôles de compétitivité par l'Etat français dans le cadre du nouveau de contrat de performance afin d'essayer d'endiguer les effets négatifs de la crise sur l'emploi : «Au départ, les pôles devaient faire de l'innovation, rien que de l'innovation. Ensuite, on nous a dit «Attention, crise oblige, les pôles vous devez être acteur des questions économiques et sociales de votre territoire. Donc, il faut intégrer cette dimension dans notre mode de fonctionnement. L'innovation c'est super, mais n'a d'intérêt qu'au service de la création de l'emploi. C'est à ce moment qu'ils ont évoqué (l'Etat) le volet formation. Donc, dans cette feuille de route, on écrit qu'il y avait un axe formation ». Une seconde source de légitimité est constituée par la cristallisation du discours, plus ou moins généralement admis, notamment par certaines entreprises du pôle, sur le rôle des RH comme facteur central de la compétitivité 
d'une entreprise. Face à ce discours, la nécessité d'intégrer une réflexion liée à la GRH au niveau du pôle de compétitivité VEGEVALLEY apparaît clairement. Trois activités vont rapidement matérialiser cette volonté : l'animation d'une association satellite développant des actions communes et regroupant les établissements de formation locaux spécialisés dans le végétal (visite d'entreprises du végétal, présentation des métiers, valorisation des jeunes diplômés dans les entreprises); l'accueil d'une des cinq semaines de formations annuelles organisées au sein de l'université européenne du végétal; le lancement des «rendez-vous stratégiques» sur le thème des RH.

\subsubsection{Importance du leadership au sein du pôle}

Le parcours professionnel de l'actuel directeur général du pôle et sa position centrale dans les réseaux d'acteurs jouent un rôle majeur dans l'animation du processus d'institutionnalisation. Après une formation d'ingénieur agronome dans une école située au cœur du territoire actuel de VEGEVALLEY, il travailla pendant 10 ans (87-96) comme directeur d'un cabinet de conseil en agriculture en collaboration étroite avec son ancienne école et les collectivités locales. Il fut alors intégré dans l'une de ces collectivités (chambre régionale d'agriculture) avant d'en devenir le directeur adjoint, fonction qu'il occupe encore aujourd'hui. Durant ces années, il fut amené à travailler sur des projets de prospective qui lui permirent de constater le sous-développement du secteur du végétal spécialisé. C'est ainsi qu'il fut nommé membre du conseil d'administration du VEGEVALLEY dès sa création en 2004. La structure de l'association du pôle a, pendant trois ans, été uniquement composée de son directeur général. Elle était physiquement hébergée dans les locaux de la chambre régionale d'agriculture. Cette dernière prit ensuite à sa charge le recrutement d'une chargée de mission pour travailler à plein temps pour le pôle. Le premier DG quitte le pôle en juin 2006 et est remplacé en juin 2007. Le second directeur restera un an puis sera remercié en juin 2008. En septembre, le conseil d'administration demande alors à l'actuel DG d'assurer l'intérim tout en conservant sa 
casquette de directeur adjoint de la chambre régionale d'agriculture. L'objectif est alors de structurer le pôle.

\subsubsection{Une structuration nécessaire et des objectifs clarifiés}

Le premier semestre 2008 correspond également au premier audit des pôles réalisé par l'Etat et dans lequel une note «moyenne» a été attribuée à VEGEVALLEY, faute de réalisations significatives. En effet, l'audit de 2008 a fait ressortir une insuffisance de projets collaboratifs et plus globalement un manque de dynamisme au sein du pôle. L'Etat n'était donc pas satisfait des orientations de VEGEVALLEY, il lui a donc proposé un nouveau contrat de performance en juillet 2009, qui a été signé fin 2009. Selon une directrice d'un cabinet de conseil en organisation et $\mathrm{RH}$, membre du pôle : «Le contrat de performance les a recadrés et VEGEVALLEY se positionne aujourd'hui sur des projets collaboratifs et l'animation d'un pôle sur du végétal en lien avec la santé, la beauté, le bien-être, respectueux de l'environnement». La dimension écologique est nouvelle pour VEGEVALLEY. Cette dimension, nouvellement imposée par l'Etat, était jusqu'à présent ignorée. Parallèlement, VEGEVALLEY a mis en place une nouvelle stratégie interne. Le pôle de compétitivité ne souhaite plus être «un réceptacle passif des idées de projets innovants qui se limite à structurer et de formaliser leur labellisation». A l'inverse, VEGEVALLEY veut être à l'origine de l'impulsion, instaurer la dynamique et prendre l'initiative devant les entreprises sur les projets innovants. «Mon prédécesseur n'avait pas les moyens. Il fallait réduire le décalage entre l'ambition et les moyens », explique l'actuel DG du pôle. Sa première action fut alors d'obtenir des ressources significatives permettant à la fois de recruter dans l'association du pôle mais également dans des associations satellitaires, sans lesquelles VEGEVALLEY n'existe pas. En 18 mois, il réussit à multiplier par 3 le nombre d'employés en passant de 8 à 25. «Ce n'est pas tant qu'il y a une prise de conscience pour donner plus de moyens, je ne suis pas sûr qu'on en avait jamais demandés. Dans le cadre du contrat de performance, moi j'ai dit, ma gouvernance c'est 2 millions d'euros, et celle des associations 
satellites doit être de 700 à 1 million chacun, ... donc c'est près de 3 millions. Aujourd'hui, on a aussi la chance d'avoir des collectivités territoriales qui ont fait du développement du végétal un axe majeur de leur politique économique, droite comme gauche. On est accompagné par les conseils généraux et régionaux, et des agglomérations du département, même modestes, mais ça reste significatif » (actuel DG du pôle).

Ces nouveaux moyens sont alors mis au service de la réalisation du nouveau contrat de performance signé avec l'Etat pour la période 2009-2012. VEGEVALLEY s'est engagé à mettre en œuvre une politique de développement répondant à deux objectifs principaux : favoriser la création et la production de végétaux favorables à la biodiversité, à l'environnement et à la santé, et ouvrir de nouveaux marchés aux entreprises du pôle grâce à des végétaux innovants. Pour réaliser ces deux objectifs, VEGEVALLEY définit plusieurs axes stratégiques : marketing, énergie-logistique, veille normative et réglementaire et $\mathrm{RH}$. Pour la majorité de ces derniers, les modalités de développement et d'organisation posaient peu problème alors que pour la dimension RH, il s'est davantage agit de gérer les contradictions, traduisant au passage la difficulté d'élaborer des représentations communes en matière de GRH. «On a alors lancé les rendez-vous stratégiques mais avec tellement peu de moyens d'animation qu'il y a eu une première séance en octobre 2008 ; une autre en janvier 2009 où on a identifié des thèmes, et on a dit : il faut qu'on avance sur cette thématique là (RH), sachant qu'entre les deux, des entreprises nous avaient fait part de leurs difficultés ».

\subsubsection{La production des textes}

On observe que le projet et les actions des acteurs ne sont que rarement linéaires, mais se chevauchent dans le temps et dans l'espace pour produire des textes nouveaux qui, s'agrégeant, participent à l'émergence de nouvelles façons de penser et d'agir. En cela, le volet $\mathrm{RH}$ de la politique de développement et de structuration du pôle a d'abord été appréhendé sous l'angle de la formation avec la création d'un bac professionnel semences 
pour répondre à un besoin manifesté à plusieurs reprises par des professionnels auprès du

Directeur du pôle. Les productions des réunions stratégiques sont les suivantes :

Tableau 3 : la production de textes

\begin{tabular}{|c|c|}
\hline Des forums RH & $\begin{array}{l}\text { Ces manifestations sont des événements relatifs à l'échange d'information } \\
\text { sur une thématique juridique, technique ou organisationnelle précise. Un } \\
\text { premier forum, organisé en septembre 2009, a été consacré au } \\
\text { renforcement des RH à l'international. Le second, prévu au } 1^{\text {er }} \text { trimestre } \\
2010 \text { a pour thème la mise en ouvre de la nouvelle loi sur l'employabilité } \\
\text { des séniors. }\end{array}$ \\
\hline Un portail RH & $\begin{array}{l}\text { Elaboration d'une plateforme informatique mutualisée de GRH (partages } \\
\text { d'expérience, offres et demandes d'emplois, travaux d'études, promotion } \\
\text { du pôle, des entreprises et de leurs métiers). Ce portail peut être utilisé par } \\
\text { tous les membres du pôle selon leurs besoins. }\end{array}$ \\
\hline $\begin{array}{l}\text { La commission } \\
\text { Emploi-formation }\end{array}$ & $\begin{array}{l}\text { Cette commission a pour objectif de mener une réflexion sur la } \\
\text { certification des emplois dans le domaine du végétal. Cette commission se } \\
\text { réunit deux fois par an et concerne les responsables de formation, les } \\
\text { entreprises en manque de main d'œuvre ainsi que les acteurs publics. Elle } \\
\text { donne lieu à diffusion à tous les membres du pôle d'un compte rendu } \\
\text { également téléchargeable sur le portail RH. }\end{array}$ \\
\hline Promotion des métiers & $\begin{array}{l}\text { Création de plaquettes et de DVD visant la communication sur les métiers } \\
\text { du végétal (types de poste, activités quotidiennes, compétences requises, } \\
\text { évolution). }\end{array}$ \\
\hline $\begin{array}{l}\text { La charte de l'emploi } \\
\text { saisonnier en } \\
\text { partenariat avec la } \\
\text { chambre d'agriculture } \\
\text { départementale, les } \\
\text { CCI, le conseil général }\end{array}$ & $\begin{array}{l}\text { Cette charte de } 6 \text { pages vise à sensibiliser aux pratiques de GRH } \\
\text { (intégration, formation, portefeuille de compétences) afin d'attirer et de } \\
\text { fidéliser les employés saisonniers qui constituent chaque année } 70 \% \text { de la } \\
\text { main-d'œuvre totale du secteur. Elle prévoit d'assurer la certification des } \\
\text { travailleurs saisonniers pour valoriser leurs compétences et leur donner } \\
\text { une reconnaissance. Cette charte fait l'objet d'une expérimentation (2010- } \\
\text { 2012) au sein de } 25 \text { entreprises choisies pour leur volonté d'avancer sur } \\
\text { ces problématiques et } 5 \text { bassins d'emplois. }\end{array}$ \\
\hline
\end{tabular}

Ces productions textuelles particulièrement objectivées amorcent la structuration d'une politique $\mathrm{RH}$ au niveau du pôle en esquissant sa forme globale, ses objectifs (attractivité, fidélisation, mutualisation de ressources, etc.) et ses niveaux d'intervention (au-delà du pôle avec la Charte ; au sein du pôle avec la promotion des métiers, portail $\mathrm{RH}$, forums ; ou une seule organisation grâce au volet d'aide au recrutement du portail RH) (Calamel, et al., 2011). Cette production renvoie directement aux spécificités du pôle. En effet, le département du Maine-et-Loire est l'un des premiers départements employeurs de salariés agricoles qui représentent $7 \%$ de la population active. C'est dans les productions liées au végétal spécialisé (arboriculture, viticulture, maraîchage, horticulture pépinières, semences...) que l'emploi salarié est le plus développé, avec la nécessité de recourir à l'embauche de salariés permanents et saisonniers pour faire face aux pics d'activité et réaliser les tâches non 
mécanisables. En 2008, les productions végétales spécialisées ont généré près de 34250 emplois, l'équivalent de 8480 temps pleins et, en 2009, trois-quarts des 46000 emplois de production agricole sont des emplois saisonniers. Le caractère cyclique de l'activité végétale amène les entreprises à augmenter leur demande de main-d'œuvre sur seulement $30 \%$ de l'année. Ce besoin intervenant à la même période pour l'ensemble des entreprises à activité saisonnière, le coût de main-d'œuvre s'accroît considérablement sur une même période. Selon une consultante RH membre du pôle, «Il n'est pas rare qu'un salarié commence sa journée sans savoir à quelle heure il la terminera. Chose qu'on ne trouverait pas dans d'autres secteurs ». Face à cette situation, une intervention politique s'impose dans la mesure où certaines entreprises souhaiteraient bénéficier d'une flexibilité totale vis-à-vis des aspects législatifs du contrat de travail. Outre la question de la reconnaissance des métiers de l'horticulture et leur valorisation, les dirigeants d'entreprises positionnent aussi VEGEVALLEY comme un levier pouvant influencer la législation sociale jugée trop contraignante par les professionnels : «L'autre aspect, c'est l'évolution de la législation sociale. C'est un problème de lobbying qu'il faut faire avancer à tous les niveaux, du syndicalisme agricole au pôle de compétitivité» (un dirigeant de coopérative horticole). Parallèlement à ces productions propres aux réunions, il est possible d'ajouter d'autres textes comme la création d'une formation sur le végétal spécialisé répondant à des besoins d'entreprises; le recrutement d'une chargée de mission pour travailler sur les questions spécifiques des RH ; le contrat de performance engageant VEGEVALLEY à agir sur les enjeux RH. On observe ainsi une production de textes multiples dont le potentiel de diffusion au sein des organisations membres apparaît variable. En effet, les supports de communication de type plaquettes et DVD faisant la promotion des métiers du végétal, la charte de l'emploi saisonnier (parce qu'elle est le fruit d'une action politique depuis plusieurs années de la chambre départementale d'agriculture), et le portail e-RH en tant qu'outil RH moderne et accessible au service des membres du pôle, constituent des textes dont la diffusion semble 
aisée. Pour autant, il reste à observer pour les productions plus récentes la manière dont les acteurs (saisonniers, employés, RRH, dirigeants) membres vont se saisir de ces outils à disposition. Ensuite, compte tenu de la présence variable des représentants d'entreprises aux rendez-vous stratégiques $\mathrm{RH}$, il est loisible de s'interroger sur le niveau de présence des dirigeants d'entreprises et autres DRH/RRH aux futurs événements créés par le pôle (comme la commission emploi-formation et les forums $\mathrm{RH}$ ) et sur leur rôle dans la diffusion de ces informations au sein de leur propres organisations. Des entretiens menés avec les dirigeants et RRH absents aux différentes réunions confirment l'idée selon laquelle ces derniers ne connaissent que faiblement l'action de VEGEVALLEY en général et les aspects RH en particulier : «le portail RH ? Vous me l'apprenez. [...] La charte de l'emploi saisonnier? C'est quoi ?» (RRH d'une coopérative agricole dont le dirigeant a participé aux réunions $\mathrm{RH}$ ).

\subsubsection{Liaison entre textes et cohérence des discours}

En revanche, les liens entres ces différents textes apparaissent assez forts, ce qui participe à la construction d'un discours gagnant en consistance et donc en puissance autour de l'attractivité des métiers du végétal, de la diffusion de bonnes pratiques RH tout autant que des obligations légales récentes en matière de GRH (les séniors, la GPEC). Cette analyse peut être nuancée par la faiblesse actuelle d'avancées significatives sur les questions de la mutualisation de l'emploi saisonnier que les professionnels considèrent comme une solution centrale à la problématique de ce type d'emploi. En définitive, parce qu'il ne peut y avoir institutionnalisation d'un discours sans le support d'autres discours, il faut maintenant analyser la structure de cohérence interne au discours RH produit et son degré de concurrence avec d'autre discours fermement ancrés au sein des membres du pôle. En effet, d'autres solutions/discours sont présents pour proposer d'autres sources de compétitivité. Parmi ces dernières, notre enquête fait apparaître trois types de discours venant «challenger» une vision «noble» des RH comme leviers de compétitivité. Ces alternatives s'incarnent à travers 
des discours sur la mécanisation, la redéfinition de la chaîne de valeur, ou les RH comme un coût à minimiser. Les citations suivantes illustrent respectivement ces trois points :

- Mécanisation : «Des producteurs de roses me disaient hier : «nous on est penché

toute la journée... ce n'est pas viable, plus personne ne veut venir. Donc travaillons sur la mécanisation pour réduire cette pénibilité. Le problème, c'est qu'avec la mécanisation, on casse les passerelles entre les filières. Puisqu'on a beaucoup de gens qui font quelque mois dans le champignon, puis en arboriculture, etc. Il y a des secteurs qui se mécanisent mais tout n'est qu'une question d'équilibre » (le directeur du pôle).

- Modification de la chaîne de valeur : «Compte tenu du coût de la main-d'œuvre, les pépiniéristes sont en train soit d'acheter des machines à rempoter, soit de le sous-traiter à des gens spécialisés qui sont équipés, mécanisés, pour faire ce genre de prestation de service. Donc, du coup, ils n'ont plus besoin des saisonniers. Pareil pour les greffons de rosiers, on a aujourd'hui des sociétés anglaises qui ont des tâcherons qui font cela à longueur de temps. Donc, du coup mes producteurs de roses, ils ont tendance à arrêter d'avoir recours à de la main-d'œuvre locale parce que c'est compliqué à former, on en n'a pas besoin longtemps, etc. » (un consultant, membre du pôle).

- Les RH comme un coût à minimiser : «On est sur une dimension des RH assez particulière de par l'activité fluctuante des entreprises ce qui entraîne la présence de beaucoup de saisonniers. On se retrouve avec des entreprises qui ont parfois tendance à avoir une attitude consommatrice, un peu mercenaire avec les $R H$. (...) On est dans quelque chose d'assez sauvage au niveau du mode de relation, pour cela il y a des choses qui sont en train d'avancer avec la Charte de l'emploi saisonnier » (une consultante, membre du pôle).

\section{DISCUSSION}

L'analyse discursive mobilisée dans cette recherche exploratoire met en lumière plusieurs résultats.

\subsection{Importance de la légitimité du producteur du texte}


Tout d'abord, l'analyse du cas met en avant la prise en compte de différents niveaux de légitimité (acteurs/textes) pour appréhender la problématique d'une GRHT. Elle montre notamment l'importance de la légitimité des porteurs de projet (Directeur du pôle, membres des réunions stratégique $\mathrm{RH}$ ) pour que les textes produits soient acceptés et puissent se diffuser. Ensuite, la mise en exergue d'une variété des dispositifs RH n'est pas sans poser de difficultés de cohérence globale en termes d'alignement vertical et horizontal. Enfin, l'analyse rend compte de l'importance du travail de traduction (Mazzilli, 2010), au sein de leurs organisations respectives, des acteurs participant aux ateliers de réflexion RH. Ces résultats permettent d'engendrer une discussion avec les travaux existants sur la GRHT et/ou des pôles autour des thèmes de la légitimité, de la variété et de la temporalité.

Concernant la question de la légitimité, Barabel, et al. (2009) avancent à la fin de leur étude que la recherche de légitimité du pôle Cosmetic Valley constitue une dimension explicative importante de son évolution. Notre travail confirme et approfondit ce point. En effet, au-delà de la recherche perpétuelle de légitimité du pôle à des fins de captation de ressources supplémentaires, cette dimension est présente à d'autres niveaux. En premier lieu, la légitimité de la question initiale n'est pas sans poser problème dans notre cas. En effet, cette question s'impose au directeur du pôle et à ses membres via le nouveau contrat de performance. Ici, la légitimité du sujet (innover en matière sociale) est imposée par l'Etat plutôt que directement perçue par les acteurs locaux, ce qui peut expliquer que les dispositifs RH manquent de cohérence textuelle finale (Phillips, et al., 2004). A l'image de notre cas, les instances de gouvernance des pôles de compétitivité ont d'abord été préoccupées par leur structuration et leur financement (Bertrand, et al., 2008) et se sont ensuite axées sur la réalisation de projets collaboratifs innovants essentiellement sur des aspects R\&D et de commercialisation. L'enjeu de la GRH est apparu bien après, comme une évidence pour certains praticiens, ou de manière plus politique pour d'autres. Deuxièmement, la légitimité du directeur du pôle (sa connaissance du secteur et la reconnaissance de son expertise dans le 
végétal) est centrale pour faire avancer les projets RH. Sa position dans le réseau, son capital social (Loubès et Bories-Azeau, 2011), sont déterminants pour identifier des axes d'innovation sociale et surtout les faire avancer. Précisons au passage qu'il s'agit là d'une piste prometteuse méritant des recherches futures même si elle a déjà fait l'objet de recherches récentes (Loubaresse, 2008).

\subsection{Les limites de la légitimité : tensions entre producteurs et récepteurs de textes}

La légitimité de la commission de réflexion RH pour porter des projets de GRH mutualisés est également en question dans la mesure où les producteurs des textes (dispositifs RH) ne sont pas les récepteurs (dirigeants d'entreprises, DRH, salariés). Dans notre recherche, la réception par les acteurs des textes produits a été évaluée par l'existence de discours RH divergents. Cette méthode fait clairement apparaître des écarts entre la structure de gouvernance qui engage des actions sur la question des $\mathrm{RH}$, et les adhérents du pôle qui ont potentiellement d'autres attentes (Bocquet et Mothe, 2008, Gomez, 2008, Mendel et Bardet, 2008). Certes, la dimension des RH à VEGEVALLEY apparaît particulièrement définie par le contexte qu'impose la filière du végétal. Cela dit, les dispositifs RH, s'ils ne sont pas si éloignés des préoccupations des acteurs, pèchent à l'heure actuelle dans leur capacité à proposer des solutions plus ambitieuses à ses adhérents notamment sur la question de la mutualisation de l'emploi ou la mise en place d'une GPEC au niveau du pôle. Cette idée doit s'inscrire dans la problématique de la détermination des négociations et des transformations conduisant à l'élaboration des dispositifs de GRHT (Mazzilli, 2011). Ainsi, VEGEVALLEY apparait bien orienté. Il engage ses premières actions sur les RH en se positionnant comme une interface d'information pour tout le milieu du végétal et pour un public plus large. La gouvernance a au final pour ambition l'amélioration de l'image négative que porte le travail dans le secteur agricole, et oriente donc une part significative de sa stratégie naissante des RH dans une optique de communication. Il s'agit là d'une piste qui pourrait minimiser les écarts d'appréciation entre les producteurs de texte et récepteurs dont 
l'efficacité mérite d'être évaluée. Dans un point de vue méthodologique, une recherche ultérieure par questionnaire pourrait objectiver quantitativement cet écart.

\subsection{Les limites de la légitimité : existence des jeux d'acteurs}

D'autres raisons expliquent cet écart et encouragent la poursuite des travaux empiriques. Avant d'engager des actions concrètes en matière RH, il s'agit pour le pôle de cadrer cette gestion spécifique sur des aspects fédérateurs qui impliquent la totalité des acteurs. A cela s'ajoutent trois facteurs qui complexifient cette élaboration de la GRH : la réalité d'adhérents non instantanément mobilisables qui freinent la dynamique de la mutualisation ; la résistance au changement qu'expriment en filigrane certains acteurs quant à l'idée d'évoluer collectivement sur la dimension des RH ; l'hétérogénéité des attentes des membres. Certaines organisations (souvent les plus petites avec peu de moyens) apparaissent en effet quelque peu «frileuses » à évoluer en termes de RH car cela est souvent uniquement synonyme, à leurs yeux, d'augmentation des coûts. D'autres solutions sont alors envisagées (mécanisation, redéfinition de la chaîne de valeur). Pour ces organisations, la question des RH n'est pas prioritaire et VEGEVALLEY est avant tout considéré comme une vitrine de communication au service des entreprises du végétal. Par ailleurs, d'autres organisations (les plus importantes en termes de taille, d'internationalisation et de ressources), ne se retrouvant pas dans les produits RH développés par le pôle (ceux précédemment évoqués), ont initiés d'autres actions sur des thématiques propres (gestion des talents, attractivités des hauts potentiels). Ce phénomène démontre, à nouveau, la difficulté de proposer une vision commune à l'ensemble des membres.

\subsection{L'approche discussive comme cadre pertinent pour saisir les difficultés de l'institutionnalisation des pratiques $\mathrm{RH}$}

Notre recherche confirme le récent travail de synthèse réalisé par Calamel et al. (2011) sur la variété des dispositifs RH attribuables tant aux éléments de contexte (problème d'attractivité et fidélisation) qu'aux jeux d'acteurs (collectivités locales avec la charte de l'emploi 
saisonnier; les groupements d'employeurs très présents veulent s'imposer comme facilitateurs de la mutualisation des pratiques de recrutement/emploi/formation voire de gestion de carrières) mais aussi au degré de facilité de la collaboration (le portail RH apparaît comme un dénominateur commun facilement acceptable par le plus grand nombre pour avancer sur ces questions de GRH au niveau du pôle). Cette variété des actions et des textes rend délicate la construction d'un discours unique et cohérent et donc une institutionnalisation des pratiques RH mutualisées difficile. Une telle situation explique les difficultés actuelles de ce pôle, et d'autres (Calamel, et al., 2011), à innover de manière significative sur le long terme en matière de RH. S'il n'est pas étonnant de constater la présence de visions RH concurrentes (mécanisation, chaîne de valeur, RH comme coûts à minimiser) à un niveau d'analyse inter-organisationnel, il nous semble que loin de considérer une telle situation comme un inconvénient, elle peut néanmoins constituer un avantage en contribuant in fine à la maturité des politiques RH au sein du pôle.

Enfin, l'étude confirme également la présence de temporalités hétérogènes (Calamel, et al., 2011) des textes produits. Ainsi, les forums RH et la création de supports de communication doivent respectivement permettre d'informer rapidement les membres du pôle sur les évolutions légales en matière sociale et d'améliorer l'attractivité des métiers au sein des entreprises du pôle. Le portail RH constitue davantage un dispositif de moyen terme devant centraliser offres et demandes d'emplois en plus de favoriser la communication sur les différentes filières métiers. La commission emploi-formation et la charte de l'emploi saisonnier apparaissent quant à eux clairement comme des dispositifs de long terme plus ambitieux.

Des suites possibles à cette enquête exploratoire consisteraient à étudier certaines pratiques RH inter-organisationnelles. Si au sein des systèmes productifs locaux, on a pu mettre l'accent sur la mutualisation pour tirer profit des démarches relatives à la main-d'œuvre ou encore le développement des engagements de développement de formation (Bories-Azeau, et al., 2007), 
au sein de VEGEVALLEY on pourrait s'intéresser à deux dispositifs RH. Il s'agit plus précisément de la création du portail RH (considéré comme une solution rapidement et facilement acceptée par la totalité des acteurs présents aux réunions) et l'expérimentation de la Charte de l'emploi saisonnier et son action sur l'amorce d'une GPEC au niveau du territoire. Ainsi, quelle que soit la forme que peuvent prendre les réseaux interorganisationnels (Ebers et Jarillo, 1998, Rorive, 2005), la question de l'efficacité des pratiques et des actions demeure au final centrale (Mahé De Boislandelle et Estève, 2007).

Les travaux en entrepreneuriat institutionnel (Basso, et al., 2009, Hatchuel, et al., 2009) pourraient constituer une grille de lecture intéressante et pertinente pour étudier la capacité d'acteurs à porter les politiques de GTEC au sein des pôles.

\section{Conclusion}

L'objectif de cette recherche était de mettre en exergue le processus d'institutionnalisation d'une politique RH au sein d'un pôle de compétitivité. D'un point de vue empirique, il semble, en l'état, délicat de considérer que les textes et les discours produits par l'action du pôle constituent une forme institutionnalisée de politique de GRH en ce sens qu'elle contraindrait l'action des acteurs et des organisations membres. Ce processus, mis en lumière grâce à notre choix théorique, apparaît néanmoins clairement en cours, même si de réelles incertitudes peuvent encore planer sur sa conclusion. Le cas met l'accent sur les pratiques discursives des acteurs ainsi que le rôle joué par les animateurs du pôle lors des ateliers RH en montrant combien la mise en cohérence de discours concurrents constitue un enjeu central pour son institutionnalisation.

Au final, cette recherche démontre, avec d'autres (Bories-Azeau, et al., 2007, Mazzilli, 2010), la difficulté de l'action collective en matière de gestion des ressources humaines interorganisationnelle. Au sein de VEGEVALLEY, la rupture n'est pas davantage consommée entre GRH traditionnelle et GRH territoriale (Calamel, et al., 2011). Pour autant, les actions en cours invitent nécessairement à la prudence du jugement quant à leurs effets dans les mois 
et années à venir. Les pôles de compétitivité, à l'image de VEGEVALLEY, n'ont finalement eu que peu d'occasion d'approfondir la dimension des RH (Bertrand, et al., 2008), certes pour des questions de temps, mais aussi de gouvernance et de divergences des représentations sur le facteur humain. La jeunesse des pôles de compétitivité amène à penser que les fruits RH semés aujourd'hui gagneront en impact demain.

\section{Références}

M. Barabel, Chabault D., Meier O. et Tixier J., "La dynamique de territoire et l'évolution d'un pôle de compétitivité: le cas de Cosmetic Valley", Management \& Avenir, 5, 25, 2009, p.144163.

O. Basso, Fayolle A. et Bouchard V., "L'orientation entrepreneuriale. Histoire de la formation d'un concept", Revue française de gestion, 195, 5, 2009, p.175-192.

H. Bertrand, Hacquemand S., Nonat P., Remond A., Seguin N., Eskl R., Leygues M. et Mayneris F. (2008). "Etude monographique sur les implications des pôles de compétitivité dans le champ de l'emploi, de la formation et des compétences.", Groupe Alpha, Cabinet GESTE, Rapport pour le Comité d'Analyse Stratégique.

R. Bocquet et Mothe C., "Gouvernance et performance des pôles de PME", Revue française de gestion, 190, 10, 2008, p.101-122.

I. Bories-Azeau, Loubès A. et Fabre C., "Emergence d'un acteur collectif territorial et réseau d'entreprises: l'exemple de CAMDIB", Revue Gestion et Management Publics, 2007,

M. Bourgain et Tixier J., "A la recherche d'un cadre d'analyse des actions RH des pôles de compétitivité", Congrès de l'AGRH, 2010, Saint Malo, France.

L. Calamel, Defélix C., Mazzilli I. et Retour D., "Les pôles de compétitivité: un point de rupture pour la GRH traditionnelle? Une analyse des dispositifs RH au sein des 12 pôles de la région Rhône-Alpes", Management \& Avenir, 1, 41, 2011, p.175-193.

R. Colle, Culié J.-D., Defélix C., Hatt F. et Rapiau M.-T., "Quelle GRH pour les pôles de compétitivité ?", Revue Française de Gestion, 2009, p.143-161.

J. Culié, Defelix C., Retour D. et Valette A., "Les pôles de compétitivité, laboratoires d'innovation en ressources humaines ?", Congrès AGRH, 2006, Reims, France.

C. Defélix, Colle R. et Rapiau M.-T., "Prendre en compte le facteur humain au sein des pôles de compétitivité : la longue marche vers l'innovation sociale.", Revue Management et Avenir, 2008, p.9-29.

C. Defélix, Dégruel M., Le Boulaire M. et Retour D., "Territorialisation de la GRH : de nouvelles démarches d'entreprise et une nouvelle GRH ?", $\mathrm{XX}^{\circ}$ Conférence de l'AGRH, 2010, Saint-Malo, France.

C. Defelix, Mazzili I., Picq T. et Retour D., "La conduite des projets collaboratifs au sein des pôles de compétitivité: l'insoutenable légèreté du management et de la GRH", Congrès de l'AGRH, 2008, Dakar, France.

M. Detchessahar et Journé B., "Une approche narrative des outils de gestion. Proposition à partir d'une étude de cas.", Revue Française de Gestion, 33, 174, 2007, p.77-92.

M. Ebers et Jarillo J. C., "The construction, forms, and consequences of industry networks", International Studies of Management and Organization, 27, 1998, p.3-21.

P.-Y. Gomez, "La gouvernance des pôles de compétitivité", Impasses théoriques et reformulation de la spécificité des pôles, 190, 10, 2008, p.197-209.

D. Grant, Hardy C., Oswick C. et Putman L. (2004) The SAGE Handbook of Organizational Discourse, London, Sage Publication Ltd, 2004.

D. Grant, Keenoy T. et Oswick C. (1998) Discourse and Organization London, Sage Publications, 1998. 
C. Hardy et Phillips N., "Discourse and power", in D. Grant, C. Hardy, C. Oswick, N. Philipps et L. Putnam (Ed.), The Sage handbook of organizational discourse, 2004, p.299316.

A. Hatchuel, Garrel G., Le Masson P. et Weil B., "L'intrapreneuriat, compétence ou symptôme? Vers de nouvelles organisations de l'innovation.", Revue française de gestion, 195, 2009, p.159-174.

E. Loubaresse, "Influence des caractéristiques des pilotes de réseaux locaux d'organisations sur leurs rôles", Management international, 13, 1, 2008, p.85-96.

A. Loubès et Bories-Azeau I., "Les réseaux territorialisés de PME: Quels effets sur la formation professionnelle continue des salariés?", Management \& Avenir, 9, 2011, p.163-175. H. Mahé De Boislandelle et Estève J., "Audit socio-économique du RLE/SPL : CAMDIB", Congrès IAS 2007, 9ème Université de printemps de l'audit social (IAS).

I. Mazzilli, "Une Gestion des ressources humaines à l'interface des organisations: vers une GRH territoriale?", AGRH, 2009, Toulouse.

I. Mazzilli, "GRH, Territoire et Traduction, ou la difficile naissance d'une gestion territoriale des ressources humaines", XX ${ }^{\circ}$ Conférence de l'AGRH, 2010, Saint-Malo, France.

I. Mazzilli, "Les dispositifs de GRH territoriale : d'une logique de transposition à la construction d'un modèle nouveau ?", XXII congrès de l'AGRH, 2011, Marrackech, Maroc. A. Mendel et Bardet M., "Quelle gouvernance pour les pôles de compétitivité constitués de PME", Revue française de gestion, 190, 10, 2008, p.123-142.

N. Phillips, Lawrence T. B. et Hardy C., "Discourse and Institutions", Academy of Management Review, 29, 4, 2004, p.635-652.

D. Robichaud, Giroux H. et Taylor J. R., "The metaconversation: The recursive property of language as a key to organizing", The Academy of Management Review, 2004, p.617-634.

B. Rorive, "L'entreprise réseau revisitée: une tentative d'ordonnancement de nouvelles formes d'organisation", Gérer et Comprendre, 79, 2005, p.63-75.

R. Stake, "Qualitative Case Studies", in N. K. Denzin et Y. S. Lincoln (Ed.), The SAGE Handbook of Qualitative Research (3rd Edition), SAGE Publications, London, 2005, p.443466.

S. Wasserman et Galaskiewicz J., Advances in social network analysis: Research in the social and behavioral sciences, Sage Publications, Inc, 1994.

R. K. Yin, Case Study Research, Design and Methods, Sage, Newbury Park, CA, 2003. 